\title{
Effect of non-surgical periodontal therapy on interleukin-29 levels in gingival crevicular fluid of chronic periodontitis and aggressive periodontitis patients
}

\author{
B.M. Shivaprasad ${ }^{\mathrm{a}, \mathrm{b}}$ and A.R. Pradeep ${ }^{\mathrm{a}, *}$ \\ ${ }^{a}$ Department of Periodontics, Government Dental College, Hospital and Research Institute, Bangalore, India \\ ${ }^{\mathrm{b}}$ Department Of Periodontics, Rajarajeshwari Dental College and Hospital, Bangalore, India
}

\begin{abstract}
Recently discovered interleukin 29 (IL-29) has antiviral properties and its production is induced by herpes viruses. This study was aimed at analyzing the effect of non-surgical periodontal treatment on IL-29 levels in gingival crevicular fluid (GCF) of chronic and aggressive periodontitis patients. A total of 60 participants were divided into healthy group (group $1 ; n=$ 20 ), chronic periodontitis group (group $2 ; n=20$ ), and aggressive periodontitis group (group $3 ; n=20$ ). GCF samples collected from each subject at baseline and 6-8 weeks after scaling and root planing were quantified for IL-29 levels using ELISA. The mean IL-29 concentration in GCF was found to be highest in group $3(92.37 \mathrm{pg} / \mu \mathrm{l})$. The mean IL-29 level in group 1 and group 2 was $36.88 \mathrm{pg} / \mu \mathrm{l}$ and $69.35 \mathrm{pg} / \mu \mathrm{l}$ respectively. After scaling and root planing, the mean concentration of IL-29 in GCF was increased to $85.99 \mathrm{pg} / \mu \mathrm{l}$ in group 2 and to $114.64 \mathrm{pg} / \mu \mathrm{l}$ in group 3. Results of the present study indicate that antiviral IL-29 level was highest in GCF of aggressive periodontitis patients and least in subjects with healthy periodontium, while that of chronic periodontitis lying in between. After non-surgical periodontal therapy, IL-29 levels increased both in chronic and aggressive periodontitis patients and deserve further investigation as a potential therapeutic agent in treating periodontitis.
\end{abstract}

Keywords: Interleukin 29, interferon lambda, chronic periodontitis, aggressive periodontitis

\section{Introduction}

Periodontal disease is a group of inflammatory disorders, primarily initiated by a chronic bacterial infection [20] and interrelated to the host response to those accumulations characterized by connective tissue breakdown and alveolar bone destruction [22,27]. A small group of predominantly gram-negative anaerobic or microaerophilic bacteria within the biofilm are often associated with disease initiation and progression. Host reactions to these pathogens result in the production of inflammatory mediators by cells like neutrophils, macrophages, T cells, mast cells and fibroblasts. Proin-

* Corresponding author: A.R. Pradeep, Department of Periodontics, Government Dental College, Hospital and Research Institute, Bangalore, India. E-mail: periodonticsgdcri@gmail.com. flammatory cytokines like Interleukin-1 (IL-1), IL6 , tumor necrosis factor (TNF- $\alpha$ ) and prostaglandins (PGE2) can promote extracellular matrix destruction by matrix metalloproteinase (MMPs) in the periodontium and stimulate bone resorption [3,23].

Cytokines play a key role in a number of biological activities including, development, proliferation, regeneration, repair and inflammation [11,26]. Contributing inflammatory mediators and tissue destructive molecules have been detected in the gingival tissues, gingival crevicular fluid (GCF) and saliva of patients affected by periodontitis. Qualitative and quantitative changes in the composition of these biomarkers could have diagnostic and therapeutic significance [18,22].

Actinobacillus actinomycetemcomitans, Porphyromonas gingivalis, and Bacteroides forsythus are considered major pathogenic species in destructive periodontal disease [32]. Some herpes viruses including Epstein- 
Barr virus type 1 (EBV-1), human cytomegalovirus (HCMV) and herpes simplex virus (HSV) have been associated with periodontal disease as well $[2,7,8]$.

A family of three cytokines, designated interleukin 29, (IL-29), IL-28A and IL-28B have been recognized from the human genomic sequence that are known as interferon- $\lambda_{1}\left(\right.$ IFN- $\left.\lambda_{1}\right)$, IFN- $\lambda_{2}$ and IFN- $\lambda_{3}$ respectively. It was also found that like type I IFNs, IL-28 and IL29 were induced by viral infection and showed antiviral activity $[9,10,19,24]$. Interferon lambda (IFN- $\lambda$ ) has antiviral, antitumour and immunomodulatory effects. Impaired IFN- $\lambda$ production may lead to increased susceptibility to viral infections [10].

Recently IFN- $\lambda$ s (IL-29, IL-28A and IL-28B) were measured by enzyme linked immunosorbent assay (ELISA) in which IL-29 levels were substantially lower in patients with chronic hepatitis $C$ than in healthy controls and patients with spontaneously resolved hepatitis [21].

The analysis of cytokine production levels is used as a tool for studying the local host response to microbial challenge $[18,12]$. IL-29 production is induced by herpes viruses and herpes viruses are present in periodontal pockets of chronic and aggressive periodontitis patients. In the light of the present knowledge the authors consider it essential to analyze and quantify the levels of antiviral IL-29 in GCF and also to observe the effect of non surgical therapy on the same.

\section{Materials and methods}

Study subjects were selected from outpatient Department of Periodontology, Government Dental College and Research Institute, Bangalore, India from March to April 2011. A total of 60 subjects ( $n=60 ; 31$ men and 29 women; age range: $21-42$ years) were divided into three groups., viz, Group 1 consisting of healthy subjects and Group 2 consisting of chronic generalized periodontitis patients and Group 3 consisting of generalized aggressive periodontitis patients.

Chronic periodontitis patients were diagnosed based on the criteria of American Academy of Periodontology classification of periodontal diseases (1999). The subjects for sampling were selected at random from individuals scheduled for a routine oral examination. Periodontal evaluation included gingival index, probing pocket depth (PPD), and clinical attachment level (CAL). All clinical measurements were performed by a single examiner. PPD and CAL were measured using a graduated William's periodontal probe.
Subjects were initially categorized into two groups based on the gingival index (GI) (Loe and Silness, 1963), PPD, CAL, and radiographic evidence of bone loss for screening purposes. Group 1 (healthy, $n=$ 20 , age range of 21-41 years, mean age of $28.55 \pm$ 5.57 years) subjects with clinically- healthy periodontium, GI $<1$ and PPD $<3 \mathrm{~mm}$, and $\mathrm{CAL}=0$, showed no evidence of bone loss based on the radiographs. Group 2 (chronic generalized periodontitis patients, $n=20$, age range of 23-42 years, mean $30.20 \pm 5.63$ years) had signs of clinical inflammation, GI $>1$, PPD $\geqslant 4 \mathrm{~mm}$ in $30 \%$ of sites, and CAL $\geqslant 1 \mathrm{~mm}$ in $30 \%$ of sites with radiographic evidence of bone loss. Group 3 (Generalized aggressive periodontitis, $n=20$, age range of 22-30 years, mean $26.10 \pm 4.23$ years), GI $>$ $1, \mathrm{PD}>6 \mathrm{~mm}$ and CAL $>3 \mathrm{~mm}$ in more than 14 teeth; at least 3 of which were not first molars or incisors with moderate to severe bone loss.

Patients with chronic generalized periodontitis (group 2) generalized aggressive periodontitis (group 3) were treated with a non-surgical approach i.e. scaling and root planing (SRP). GCF samples were collected from the same sites six to eight weeks after the treatment.

All patients were systemically healthy and had not received periodontal treatment or antibiotics for at least 6 months prior to the clinical examination and sampling. Patients were excluded from the study if they had diabetes, smoking habit, any other systemic disease or conditions such as pregnancy that could alter the course of periodontal disease. Subjects who satisfied the inclusion criteria of the study were selected and ethical approval was obtained from the institutional review board. Furthermore, each patient received a detailed explanation regarding the study procedure, and written informed consent was obtained from those who agreed to participate voluntarily in the study.

\section{Site selection and GCF sample collection}

In the periodontitis group, only one site per subject was selected as a sampling site; whereas in the healthy group, multiple sites (three to five sites per subject) with an absence of inflammation were sampled to ensure the collection of an adequate amount of GCF [13]. In chronic and aggressive periodontitis patients, sites showing the greatest CAL and signs of inflammation, along with radiographic bone loss were selected for sampling using graudated Williams periodontal probe. On the subsequent day, the sample collection site was 


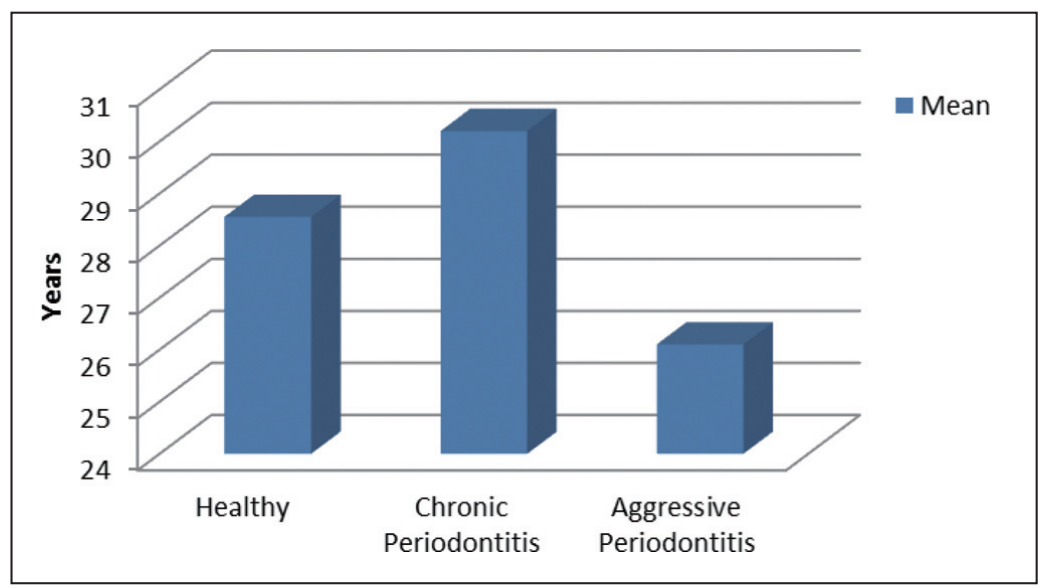

Fig. 1. Mean age in different groups. (Colours are visible in the online version of the article; http://dx.doi.org/10.3233/DMA-120944)

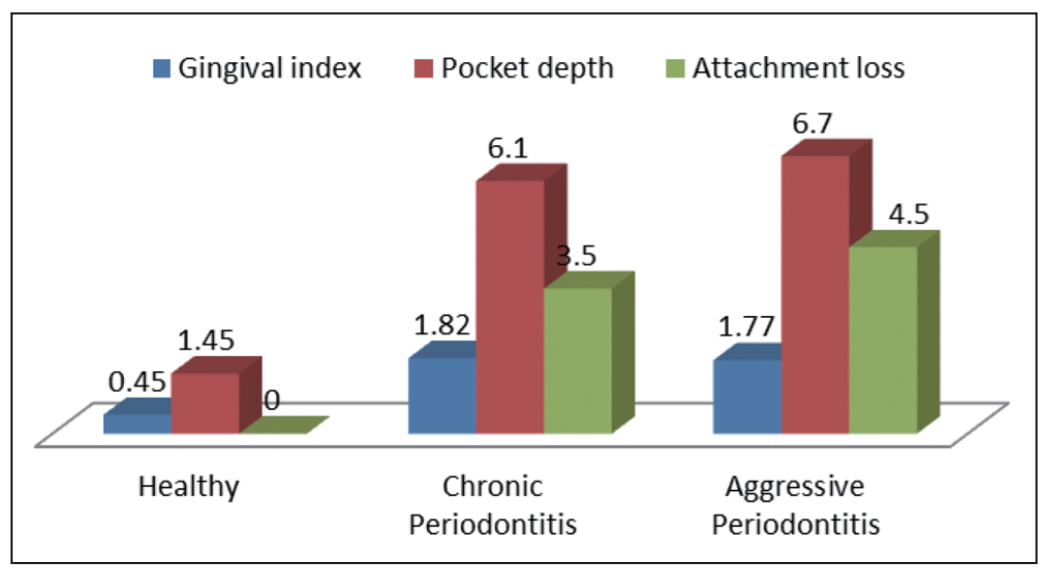

Fig. 2. Mean Gingival Index, Pocket depth and Attachment Loss in millimeters. (Colours are visible in the online version of the article; http:// dx.doi.org/10.3233/DMA-120944)

well-isolated and without touching the marginal gingiva, the supragingival plaque was removed. GCF was collected by placing the microcapillary pipette (SigmaAldrich, St. Louis, MO) at the entrance of the gingival sulcus (extrasulcular method) by gently touching the gingival margin. A standardized volume of $3 \mu \mathrm{L}$ was collected in single capillary from the same site using the calibration on white color coded 1 to $5 \mu \mathrm{L}$ calibrated volumetric microcapillary pipettes. A maximum of ten minutes was given for each sample collection, and the sites that did not express any GCF within the allotted time were excluded. The micro-pipettes that were suspected to be contaminated with blood and saliva were excluded from the study. The collected GCF samples were transferred to airtight plastic vials and stored at $-70^{\circ}$ until assayed.

\section{IL-29 assay}

IL-29 levels in GCF, obtained from study participants, were measured using Human IL-29 ELISA according to the manufacturer's instructions (R\&D Systems, USA, Imported by Biotech-India, India). All steps were performed carefully as per the guideline protocol provided by the manufacturer. The absorbance of each well is read on an ELISA reader using 450 $\mathrm{nm}$ as the primary wavelength. The concentrations of IL-29 in both the GCF and plasma tested samples were estimated using the standard curve.

\section{Statistical analyses}

All data were analyzed using a software program (STATA Version 9.2 (STATA corp LP, College Station, 
Table 1

Comparison of three groups with respect to IL-29 level in GCF by one way ANOVA

\begin{tabular}{lccccc}
\hline Description of groups & Degrees of freedom & Sum of squares & Mean sum of squares & F-value & p-value \\
\hline Between groups & 2 & 31091.5241 & 15545.7621 & 10.5968 & $0.0001^{*}$ \\
Within groups & 57 & 83620.3681 & 1467.0240 & & \\
Total & 59 & 114711.8922 & & & \\
\hline${ }^{*} p<0.05$, significant. & & & & &
\end{tabular}

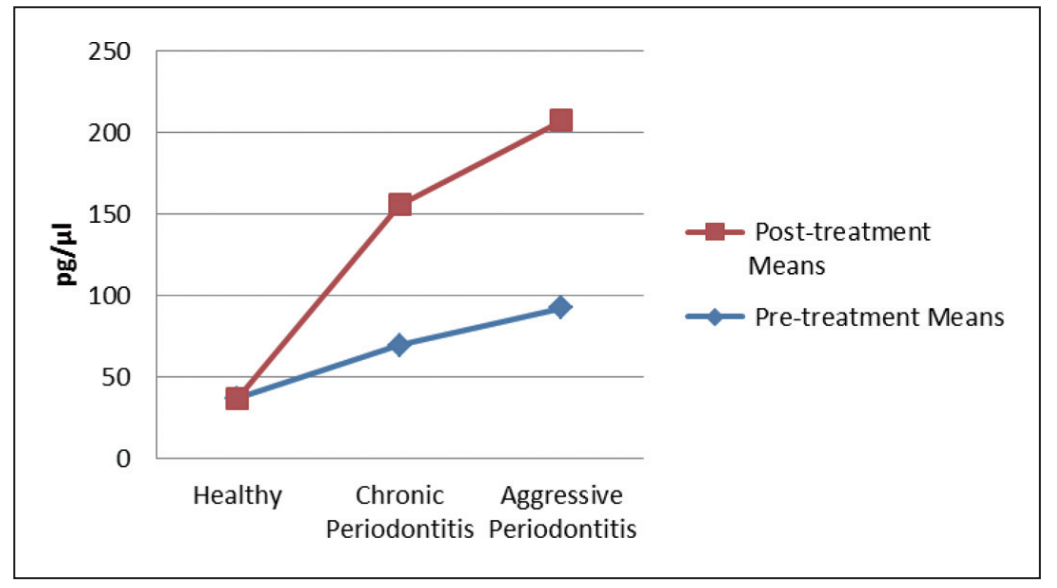

Fig. 3. Mean concentration of IL-29 in different groups. (Colours are visible in the online version of the article; http://dx.doi.org/10.3233/ DMA-120944)

TX, USA). Accordingly, parametric tests were carried out for comparing the means of IL-29 concentration in different groups. To test the hypothesis of equality of means among the four groups, ANOVA was carried out. Paired ' $t$ ' test was used to compare post treatment IL-29 concentrations in GCF of group 2 and group 3 patients. Pair-wise comparison using Tukeys test for GCF IL-29 was carried out to explore which pair or pairs differ significantly at 5\% level of significance. Pearson's correlation test was used to observe any correlation between the GCF IL-29 concentration and clinical parameters.

\section{Results}

The mean Interleukin-29 (IL-29) concentration in GCF was found to be the highest in group 3, i.e. $92.37 \mathrm{pg} / \mu \mathrm{l}$. The mean IL-29 level in group 1 and group 2 was $36.88 \mathrm{pg} / \mu \mathrm{l}$ and $69.35 \mathrm{pg} / \mu \mathrm{l}$ respectively. Post treatment the mean concentration of IL-29 in GCF increased to $85.99 \mathrm{pg} / \mu \mathrm{l}$ in group 2 and to $114.64 \mathrm{pg} / \mu \mathrm{l}$ in group 3. The mean concentration and range of IL-29 in all groups is shown in Fig. 3. Figure 1 shows the mean age and standard deviation of study subjects in different groups and Fig. 2 illustrates the mean gingival index, pocket depth and attachment loss in millimeters.
ANOVA indicated that the means differ significantly among the groups $(p<0.0001)$ (Table 1). Further post hoc analysis using Tukey's test showed that the differences were statistically significant between groups 1 and 2, groups 2 and 3 and groups 1 and $3(p<0.05)$ (Table 2).

IL-29 levels were analyzed in group 2 and group 3 six to eight weeks after scaling and root planing using paired ' $t$ ' test. The difference in the GCF IL-29 levels before and after scaling was found to be statistically significant in both chronic and aggressive periodontitis groups. IL-29 levels increased significantly (Statistically) after scaling and root planing (Table 3 ).

Pearson's correlation coefficient test was carried out to find correlation between clinical parameters, i.e. GI, PPD, CAL and IL-29 concentration in GCF. It showed a significant positive correlation between IL-29 concentration and clinical parameters in groups 2 and group 3 (Table 4).

\section{Discussion}

To the best of our knowledge, this is the first study conducted in any population regarding the detection of IL-29 in GCF of subjects with healthy periodontium, 
Table 2

Pair wise comparison of three groups with respect to IL-29 level in GCF by Tukeys multiple post hoc procedures

\begin{tabular}{lccc}
\hline Groups & Healthy & Chronic periodontitis & Aggressive periodontitis \\
\hline Mean & 36.88 & 69.35 & 92.37 \\
SD & 25.12 & 40.93 & 45.77 \\
Healthy & - & & \\
Chronic Periodontitis & $0.0257^{*}$ & $0.0002^{*}$ & - \\
Aggressive Periodontitis & $0.0002^{*}$ & & \\
\hline
\end{tabular}

${ }^{*} p<0.05$, significant.

Table 3

Comparison of before and after treatment with respect to IL-29 level in GCF in chronic and aggressive periodontitis group by paired t-test

\begin{tabular}{|c|c|c|c|c|c|c|c|}
\hline Groups & Treatment & Mean & SD & Mean Diff. & SD Diff & Paired t & P-value \\
\hline \multirow[t]{2}{*}{ Chronic periodontitis } & Before & 69.3450 & 40.9262 & & & & \\
\hline & After & 85.9865 & 49.1615 & -16.6415 & 18.1188 & -4.1075 & $0.0006^{*}$ \\
\hline \multirow[t]{2}{*}{ Aggressive periodontitis } & Before & 92.3735 & 45.7745 & & & & \\
\hline & After & 114.6380 & 56.8647 & -22.2645 & 16.2816 & -6.1155 & $0.0000^{*}$ \\
\hline
\end{tabular}

${ }^{*} p<0.05$, significant; $\mathrm{SD}=$ Standard Deviation.

Table 4

Karl Pearson's correlation coefficients between IL-29 level in GCF with Gingival index, Pocket depth and Attachment loss in (total) all groups

\begin{tabular}{cccc}
\hline Variables & Correlation coefficients between IL-29 level in GCF with & \\
\cline { 2 - 4 } & Correlation coefficient & t-value & p-value \\
\hline Gingival index & 0.5138 & 4.5606 & $0.0000^{*}$ \\
Pocket depth & 0.6747 & 6.9617 & $0.0000^{*}$ \\
Attachment loss & 0.7156 & 7.8011 & $0.0000^{*}$ \\
\hline
\end{tabular}

${ }^{*} p<0.05$ (Significant).

chronic periodontitis and aggressive periodontitis patients. Interleukin-29 levels were analyzed using sandwich ELISA technique.

Periodontal diseases are initiated by Gram-negative tooth associated microbial biofilms that elicit a host response, with resultant osseous and soft tissue destruction. Many studies have already proven the existence of herpes viruses in periodontal pockets/tissues of chronic and aggressive periodontitis patients $[2,7,8]$.

The production of IL-29 is both increased and decreased by the herpes viruses. The active role of herpes virus in periodontal diseases is debatable and not been established consistently. Immunologic response of the host by producing antiviral molecules like IL29 might be one of the mechanisms of counteracting herpes viruses in periodontal pockets/tissues.

In this study we analyzed the GCF levels of IL-29 as a biomarker for periodontal health, chronic and aggressive periodontitis. Also the effect of non surgical periodontal therapy on the IL-29 levels in GCF of periodontal pockets was assessed using ELISA technique.

Interleukin-29 is an established molecule for its anti herpes viruses activity in other organs and tissues. In a study conducted by Langhans et al. [21], IFN-lambda1 (IL-29) and IFN-lambda2/3 (IL-28A/B) plasma levels in patients with different outcomes of $\mathrm{HCV}$ infection were analyzed using ELISA. IL-29 levels were substantially lower in patients with chronic hepatitis $\mathrm{C}$ than in healthy controls $(p=0.005)$ and patients with spontaneously resolved hepatitis $(p=0.001)$. Patients with acute hepatitis $\mathrm{C}$ showed IL-29 levels intermediate between chronic hepatitis $\mathrm{C}$ and normal controls; and IL-29 plasma levels were higher in patients who spontaneously resolved hepatitis $\mathrm{C}$ than in those who became chronic. High IFN-lambda levels predisposed to spontaneous resolution of HCV infection. Thus, IFNlambdas seem to play an important role in the control of viral disease (hepatitis $\mathrm{C}$ ).

In our study IL-29 levels were found highest in aggressive periodontitis patients $(92.37 \pm 45.77 \mathrm{pg} / \mu \mathrm{l})$ and least in periodontally healthy subjects $(36.88 \pm$ $25.12 \mathrm{pg} / \mu \mathrm{l})$, while the levels in chronic periodontitis patients $(69.35 \pm 40.93 \mathrm{pg} / \mu \mathrm{l})$ were in between aggressive periodontitis patients and healthy subjects. IFNlambdas are expressed in virus-infected cells and induce marked antiviral protection in a wide variety of cells $[1,21]$. This finding suggests that increased levels of IL-29 found in aggressive periodontitis may be due to the increased prevalence of herpes viruses in the periodontal pockets compared to that of chronic periodontitis. A previous study had shown that herpes viruses like human cytomegalovirus and Epstein-Barr viruses 
were found more in periodontal pockets of aggressive periodontitis than in that of chronic periodontitis and healthy subjects [17]. IL-29 is primarily known for its expression in potentially any cell type after viral infection [33].

Also, the IL-29 level in GCF of periodontitis patients was higher than in that of healthy subjects. A previous study had shown that prevalence of HCMV and HSV viruses in GCF is higher in patients suffering from periodontitis compared to periodontally healthy subjects [14]. Considering the results of both studies it can be inferred that IL-29 level was higher in periodontitis patients because of increase in herpes viruses in periodontal pockets of chronic and aggressive periodontitits patients when compared to that of periodontally healthy subjects.

In a previous study it was shown that HSV-1 both induces and inhibits the antiviral response in human cells and that the type III IFN (IL-29), together with IFN-alpha, amplifies the antiviral response against the viruses [25]. Also, the data suggest a novel role for IL-29 in the antiviral response against HSV-1, suggesting a potential therapeutic role for type III IFNs against HSV infections. Authors here hypothesize that elevated levels of IL-29 in periodontitis patients can be reasoned because of the induction of IL-29 production by increased presence of herpes viruses in periodontal pockets compared to that of subjects with healthy periodontium. It might be the mechanism of the host to counteract the presence of herpes viruses.

Herpes viruses are present (in less number) even in the clinically healthy periodontium with shallow gingival sulcus and it is proven by previous research [29]. In the light of the above finding we can understand the reason for detecting low levels of IL-29 in GCF of subjects with healthy periodontium.

Further, scaling and root planing aimed at arresting periodontitis progression resulted in statistically significant enhancement in the levels of IL-29 in GCF. A previous study had revealed the decrease in the prevalence and also the elimination of herpes viruses after scaling and root planing [14]. Here we hypothesize that increase in the IL-29 level after scaling and root planing might be because of the decrease in herpes viruses after scaling and root planing. Also in a study conducted by Langhans et al. it was observed that IL-29 levels were substantially lower in patients with chronic hepatitis $\mathrm{C}$ than in healthy controls $(p=0.005)$ and patients with spontaneously resolved hepatitis [21]. These data suggest that as the virus level is decreased the level of the interleukin-29 increases or vice versa is true.
Also, scaling and root planing decrease the pathogenic anaerobic bacteria that coexist with herpes viruses which can increase the local host immunity [6]. Increase in local immunity can increase the IL-29 level which is antiviral and act against herpes viruses.

In the present study the levels of IL-29 correlated positively with gingival index, pocket depth and attachment loss. Previous studies have shown that increased pocket depth correlates with increased prevalence of herpes viruses and therefore we reason here that IL-29 levels increases as the pocket depth increases. After scaling and root planing the same trend of increase in IL-29 levels was seen. Also it is very important to note that Herpes viruses both induces and decreases the production of IL-29 [25]. Here authors hypothesize that presence of herpes viruses might induce the production of IL-29 in periodontal pockets. Scaling and root planing increase the local immunity in periodontal pockets which might increase the IL-29 production.

Microcapillary pipettes were used to collect GCF to avoid nonspecific attachment of the analyte, which is seen with filter paper fibers resulting in false decrease in the detectable IL-29 levels that in turn can lead to miscalculation of the correlation of IL-29 levels to disease severity. The disadvantage of this method is the possibility of trauma to the marginal gingiva, but utmost care was taken to avoid this during GCF collection [13, $15]$.

IL-29 was not detected in all the samples of GCF. The reason for this finding might be due to the variation in the sampling and also might be due to the sensitivity of the kit used. Future studies done on large sample size would better enlighten the presence of IL-29 in GCF of patients with periodontal diseases.

The limitations of the present study are 1) Other antiviral interleukins like IL-28A and IL-28B and other systemic markers are not analyzed 2) Herpes virus count was not correlated with IL-29 levels in GCF and plasma. Previous studies conducted by many researches provide insight about the onset and progression of periodontitis $[4,5,16,28,31]$. Indicators of inflammation may be important clinical determinants of future periodontal disease progression [30].

\section{Conclusion}

Results of the present study indicate that IL-29 is detected in GCF of healthy, chronic and aggressive periodontitis patients. The levels of IL-29 is highest in GCF of aggressive periodontitis patients and least in subjects 
with healthy periodontium and whereas that of chronic periodontitis lies in between. After non-surgical therapy the IL-29 levels increased in both chronic and aggressive periodontitis patients and deserves further consideration as a therapeutic agent. Future studies with larger sample size will enhance the knowledge of the role of IL-29 in periodontal health and disease.

\section{References}

[1] N. Ank, M.B. Iversen, C. Bartholdy et al., An important role for type III interferon (IFN-lambda/IL-28) in TLR-induced antiviral activity, J Immunol 180 (2008), 2474-2585.

[2] S. Bilichodmath, S.B. Mangalekar, D.C. Sharma et al., Herpesviruses in chronic and aggressive periodontitis patients in an Indian population, J Oral Sci 51 (2009), 79-86.

[3] H. Birkedal-Hansen, Role of cytokines and inflam-matory mediators in tissue destruction. J Periodontal Res 28 (1993), 500510.

[4] P. Bullon, M.D. Cordero, J.L. Quiles et al., Mitochondrial dysfunction promoted by Porphyromonas gingivalis lipopolysaccharide as a possible link between cardiovascular disease and periodontitis, Free Radic Biol Med 50(10) (2011), 1336-43.

[5] P. Bullon, J.M. Morillo, M.C. Ramirez-Tortosa et al., Metabolic syndrome and periodontitis: is oxidative stress a common link? J Dent Res 88(6) (2009), 503-18.

[6] M. Chalabi, F. Rezaie, S. Moghim et al., Periodontopathic bacteria and herpesviruses in chronic periodontitis, Mol Oral Microbiol 25(3) (2010), 236-40.

[7] A. Contreras, J. Slots, Mammalian viruses in human periodontitis, Oral Microbiol Immunol 11 (1996), 381-386.

[8] A. Contreras, H. Nowzari, J. Slots, Herpesviruses in periodontal pocket and gingival tissue specimens, Oral Microbiol Immunol 15 (2000), 15-18.

[9] K. Domagalski, A. Tretyn, M. Pawłowska, J. Szczepanek and W. Halota, Action of type III IFNs and their roles in immune responses, Postepy Hig Med Dosw 64 (2010), 522-33.

[10] R.P. Donnelly, S.V. Kotenko, Interferon-lambda: A new addition to an old family, J Interferon Cytokine Res 30(8) (2010), 555-64.

[11] K. Erciyas, S. Pehlivan, T. Sever et al., Association between TNF- $\alpha$, TGF- $\beta 1$, IL- 10 , IL- 6 and IFN- $\gamma$ gene polymorphisms and generalized aggressive Periodontitis, Clin Invest Med 33(2) (2010), E85-E91.

[12] R. Genco, Host responses in periodontal diseases: Current concepts, J Periodontol 63 (1992), 338-355.

[13] G. Garg, A.R. Pradeep, Manoj Kumar Thorat, Effect of nonsurgical periodontal therapy on crevicular fluid levels of Cathepsin K in periodontitis, Arch Oral Biol 54 (2009), 10461051 .

[14] G. Grenier, G. Gagnon, D. Grenier, Detection of herpetic viruses in gingival crevicular fluid of patients suffering from periodontal diseases: Prevalence and effect of treatment, Oral Microbiol Immunol 24(6) (2009), 506-9.

[15] G.S. Griffith, Formation, collection and significance of gingival crevicular fluid, Periodontol 200031 (2003), 32-42.

[16] C. Hewitt, D. McCormick, G. Linden et al., The role of cathep$\sin \mathrm{C}$ in Papillon-Lefèvre syndrome, prepubertal periodontitis, and aggressive periodontitis, Hum Mutat 23(3) (2004), 222-8.
[17] A.V. Imbronito, O.S. Okuda, N. Maria de Freitas et al., Detection of herpesviruses and periodontal pathogens in subgingival plaque of patients with chronic periodontitis, generalized aggressive periodontitis, or gingivitis, J Periodontol 79 (2008), 2313-2321.

[18] J. Kamma, A. Mombelli, K. Tsinidou, V. Vasdekis and C. Gianno-poulou, Cytokines in gingival crevicular fluid of adolescents and young adults, Oral Microbiol Immunol 24(1) (2009), 7-10.

[19] C. Kelly, P. Klenerman and E. Barnes, Interferon lambdas: The next cytokine storm. Recent advances in basic science, Gut 60 (2011), 1284-1293.

[20] M.A. Listgarten, Pathogenesis of periodontitis, J Clin Periodontol 13(5) (1986), 418-430.

[21] B. Langhans, B. Kupfer, I. Braunschweiger et al., Interferonlambda plasma levels in hepatitis C, J Hepatol 54(5) (2011), 859-865.

[22] C.S. Miller, C.P. King, M.C. Langub, R. Krysico, V.M. Thomas, Salivary biomarkers of existing periodontal disease. A cross sectional study, J Am Dent Assoc 137 (2006), 322-9.

[23] ManojKumar Thorat, A.R. Pradeep, G. Garg, Correlation of Levels of Oncostatin M Cytokine in Crevicular Fluid and Serum in Periodontal Disease, Int J Oral Sci 2(4) (2010), 198-207.

[24] M. Li, X. Liu, Y. Zhou, S. Bo Su, Interferon- $\lambda$ s: the modulators of antivirus, antitumor, and immune responses, $J$ Leukoc Biol 86 (2009), 23-32.

[25] J. Melchjorsen, J. Siren, I. Julkunen, S.R. Paludan, S. Matikainen, Induction of cytokine expression by herpes simplex virus in human monocyte-derived macrophages and dendritic cells is dependent on virus replication and is counteracted by ICP27 targeting NF-kB and IRF-3, J Gen Virol 87 (2006), 1099-1108.

[26] H. Okada, S. Murakami, Cytokine expression in periodontal health and disease, Crit Rev Oral Biol Med 9 (1998), 248-66.

[27] S. Oswal, C.D. Dwarkanath, Relevance of gingival crevicular fluid components in assessment of periodontal disease - A critical analysis, J Indian Soc Periodontol 14(4) (2010), 282286.

[28] M. Ricci, F. Garoia, C. Tabarroni et al., Association between genetic risk score and periodontitis onset and progression: A pilot study, Arch Oral Biol 56(12) (2011), 1499-505.

[29] I. Saygun, S. Sahin, A. Ozdemir, Detection of herpes viruses in patients with chronic periodontitis and the relationship between viruses and clinical parameters, J Periodontol 73 (2002), 1437-1443.

[30] P. Stashenko, T. Van Dyke, P. Tully et al., Inflammation and genetic risk indicators for early indicators for early periodontitis in adults, J Periodontol 82(4) (2011), 588-96.

[31] J. Suzuki, N. Aoyama, M. Ogawa et al., Periodontitis and cardiovascular diseases, Expert Opin Ther Targets 14(10) (2010), 1023-7.

[32] A. J. Van Winkelhoff, B.G. Loos, W.A. Van Der Reijden and U. Van Der Velden, Porphyromonas gingivalis, Bacteroides forsythus and other putative periodontal pathogens in subjects with and without periodontal destruction, J Clin Periodontol 29 (2002), 1023-1028.

[33] K. Wolk, K. Witte, E. Witte et al., Maturing dendritic cells are an important source of IL-29 and IL-20 that may cooperatively increase the innate immunity of keratinocytes, J Leukoc Biol 83 (2008), 1181-1193. 


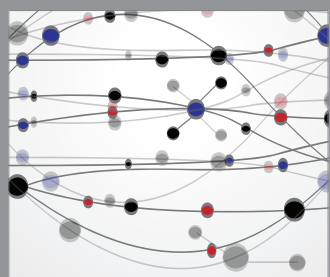

The Scientific World Journal
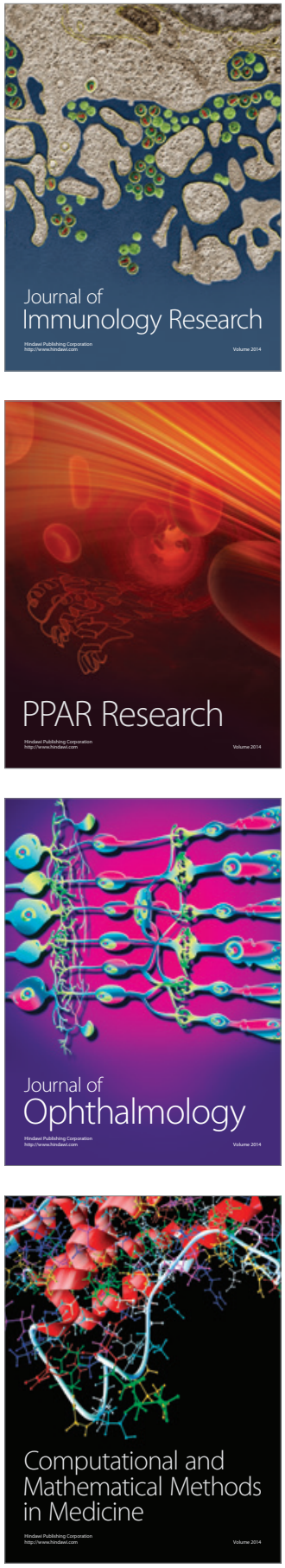

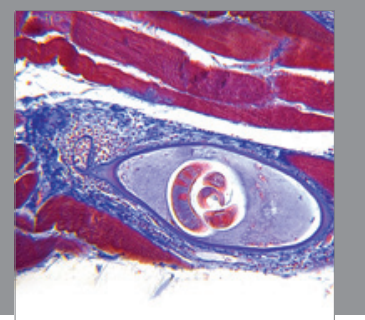

Gastroenterology

Research and Practice
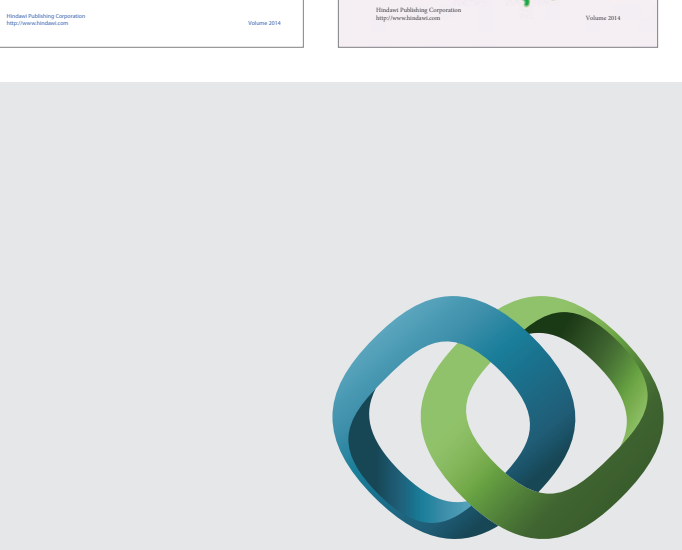

\section{Hindawi}

Submit your manuscripts at

http://www.hindawi.com
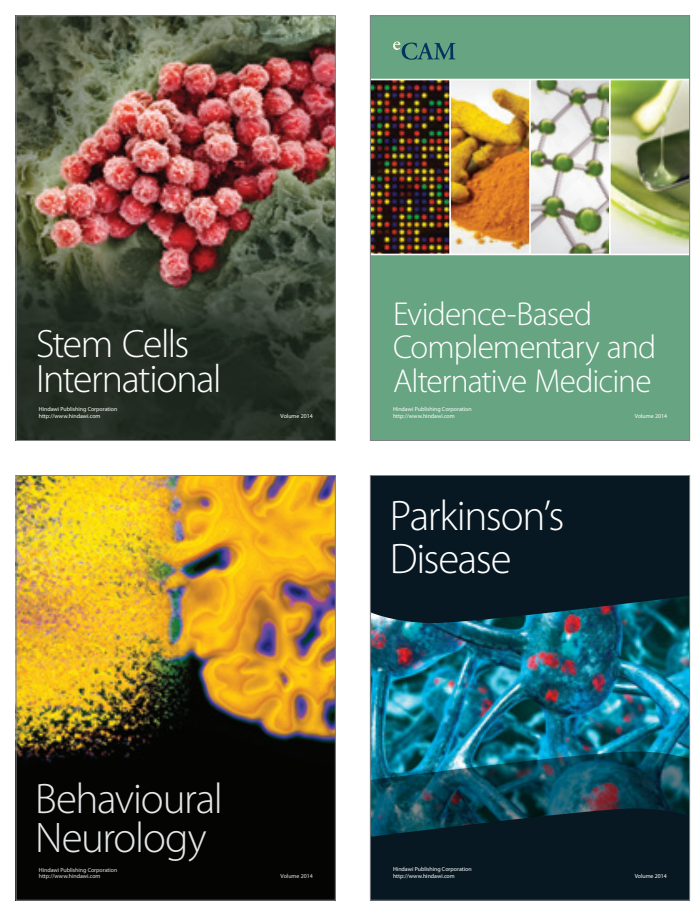

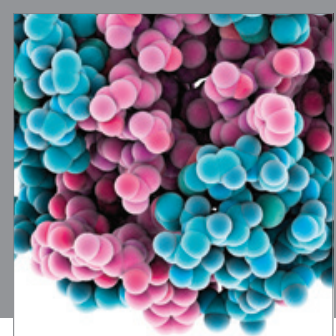

Journal of
Diabetes Research

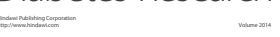

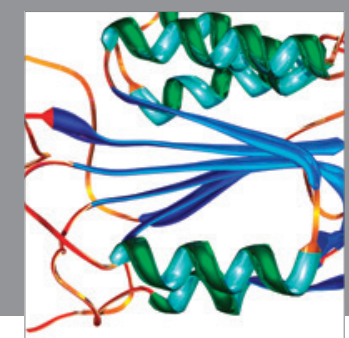

Disease Markers
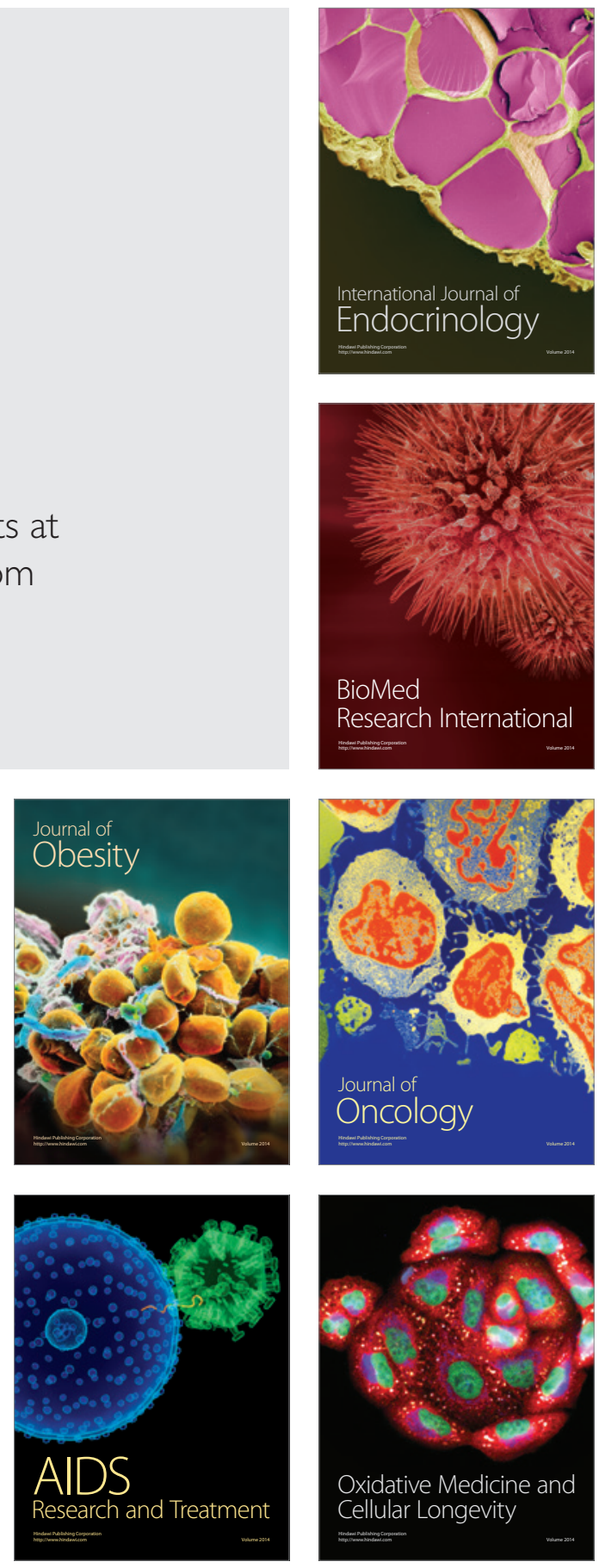